\title{
Visual Expression of Volumetric Smoke Animation by Dynamic Luminance Information
}

\author{
Yusuke Chiba and Takafumi Koike* \\ Hosei University, 3-7-2 Kajino-cho, Koganei-shi, Tokyo and 184-8584, Japan \\ *Corresponding Author: takafumi@hosei.ac.jp
}

\begin{abstract}
We present a method to create animations to express movement of volumetric smoke by compositing still images of computer graphics (CG) with a dynamic luminance information (DLI). Volume rendering is one of the rendering methods considering absorption and scattering of light by a medium involved in the air such as smoke. It can express smoke more realistic than normal rendering, but it takes a lot of time to render smoke CG animations. Thus, we express smoke motion of the animation created using volume rendering by combining a still image by normal rendering with DLI. We have experimented to compare perceptual quality of animations created by our method and normal volume rendering. We use image quality evaluation index and questionnaire for evaluation of perceptual quality. Experiment results show that our method can shorten the creation time and create animations keeping perceptual quality of animations using volume rendering.
\end{abstract}

Keywords: computer graphics, volume rendering, smoke animation; dynamic luminance information

\section{Introduction}

Computer graphics (CG) is a method to create a three-dimensional image or video from objects in virtual three-dimensional space. In recent years, CG is widely used in the field of cinemas and games.

There are two major methods of CG creation: pre-rendering and real-time rendering. Pre-rendering is mainly used to produce realistic images such as movies but consumes a long time for rendering. On the other hand, real-time rendering is used for games and interactive applications, and CG images are generated in response to user operations and inputs. While images can be generated in real time, due to differences in computational complexity, realistic representations and image accuracy are inferior to pre-rendering.

Volume rendering is a rendering technique which takes into account absorption and scattering of light in participating media such as smoke ${ }^{(1)}$ and clouds. While volume rendering is possible to represent participating media realistically, it is not suitable for real-time rendering because the computation time is very long.

On the other hand, human beings may feel movement from still images due to illusion. Kawabe et al. have proposed illusionary motion to still images by projecting dynamic luminance information (DLI) onto a single still image or real object ${ }^{(2)}$. However, the target of projection is only a still image of the real world, not a CG image.

Based on this idea, we have proposed a method of creating an illusive smoke animation by overlaying DLI on a CG still image ${ }^{(3)}$. By compositing the DLI with the normal rendering CG scene, we expressed smoke animation close to an animation rendered by volume rendering. In addition, from the experiment using a pair of images, we have obtained a result that our method is effective to give the impression of smoke.

In this paper, we firstly describe our method presented in our previous paper ${ }^{(3)}$, and we prepare a plurality of images created by our method and images rendered by volume rendering. We also experiment to compare perceptual quality and investigate the effectiveness of our method. In addition, we aim to create an image that preserves the perceptual quality of the image using volume rendering while shortening the creation time of the CG image with smoke.

\section{Related Works}

CG image generation is divided into three steps: mod- 
eling, animation generation, and rendering. In modeling, we create an object that is three-dimensional shape data. Animation generation represents the motion of an object. In rendering, data from modeling and animation generation is used to generate an image viewed from a camera in virtual space. When fluid such as smoke is generated as CG image, modeling and animation generation are mainly performed using fluid simulation. The result is rendered, and the final image is obtained.

As a method of expressing motion without using fluid simulation, there is a method of performing specific processing on a still image. In the field of image-based rendering, motion is expressed in a still image by using a fluid image database for a two-dimensional still image. In the field of projection mapping, human visual characteristics are used to express illusionary motion. By projecting the movement of brightness on a real-world still image, we reproduced the flicker of a candle and water.

\subsection{Expression of Movement by Fluid Simulation}

The motions of various fluids such as water and smoke that exist in nature are often expressed by physical fluid simulation. One of the typical fluid simulation methods is to solve the Navier-Stokes equation. The Navier-Stokes equation is an equation that describes the motion of a fluid in fluid mechanics and represents the conservation law of momentum flow in Newtonian mechanics. There are time terms, advection terms, pressure terms, viscosity terms, and external force terms in the equation. By substituting values into each term, the movement of the fluid with time is obtained.

Fedkiw et al. have presented a method to simulate the movement of smoke by solving the Navier-Stokes equation while applying a virtual force as an external force ${ }^{(4)}$. By considering the virtual force as an external force term, the numerical error of the solution of the equation is suppressed, and the smooth smoke movement can be expressed.

Chern et al. have presented a smoke representation method using Schrödinger equation ${ }^{(5)}$. Schrödinger equation is a partial differential equation mainly used in the field of quantum mechanics. The solution of the equation is called the wave function and represents the state of the quantum system such as electrons. We also describe the temporal change of the state of the quantum system. By applying this wave function to fluid simulation, the complex motion of smoke can be stably represented.

While both methods can generate smooth smoke movement, they require complex simulations and renderings, and they take time to create images.

\subsection{Expression of Movement of Still Images}

Okabe et al. have presented a system that gives water movement to a selected area in a still image, using a database of fluid images obtained by taking pictures of water flow $^{(6)}$. For the selected area, they select the most appropriate fluid image from the database and synthesize the still image to reproduce the smooth water movement. While smooth images can be obtained, it is necessary to prepare a large amount of fluid image data to create a database.

Kawabe et al. have presented a light projection technology "HenGenTou" that expresses motion by projecting DLI to a still image of a real world object by a projector ${ }^{(2)}$. Generally, in human visual information processing, an object is perceived by integrating three elements of color, shape, and motion in the brain. On the other hand, in visual science, there is illusionary information in which a stationary object appears to move due to the influence of surrounding motion information. The "HenGenTou" creates an illusion based on DLI, and makes the still image appear as if it were moving.

Among the representation methods of fluid movement, we focus on the "HenGenTou" which has low calculation cost and little preparation. In "HenGenTou", motion is expressed by projecting DLI on a real-world still image, but there has been no effective applications for CG still images. In this study, we create a smoke image by combining DLI with CG still images. We also investigate the difference between the created animation and the volume rendering animation from the aspects of quantitative evaluation and subjective evaluation.

\section{Preliminary: Volume Rendering}

At present, ray tracing is one of the mainstream $C G$ rendering methods. In ray tracing, the reflection and refraction of light emitted from a light source are traced, and the color and brightness of the light incident on the camera are determined. Ray tracing normally assumes a vacuum state, so it can't render many phenomena.

For example, a phenomenon called air perspective, in which the more distant things look darker, occurs when light that reaches the viewer from a distant thing is scattered in the atmosphere. The phenomenon of emitting light, such as a substance that acts on light without a surface or a flame, is also difficult to express under vacuum. 


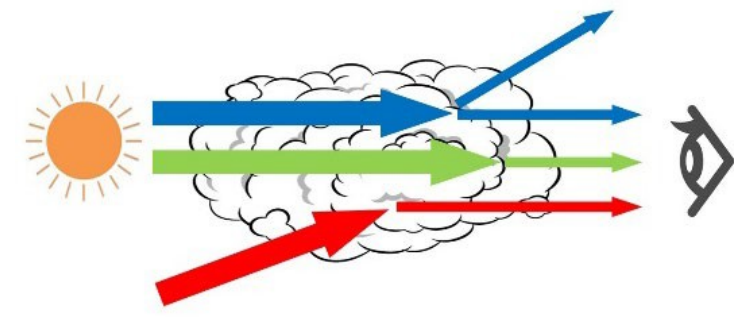

Fig. 1. Concept of volume rendering

Volume rendering is a rendering technique that takes into account the absorption and scattering of light by the participating media such as smoke. The behavior of light is calculated by solving the volume rendering equation ${ }^{(7)}$. The equation represents the amount of light change $d L(x, \vec{\omega})$ as it travels a small distance in the direction $\vec{\omega}$ towards a certain position $x$ in the medium. The amount of light change depends on the phenomenon that occurs in the medium, so the equation for each phenomenon is shown below. Fig. 1 shows a concept of volume rendering.

\subsection{Light Attenuation in Participating Media}

As shown by an arrow on the top of Fig. 1, when light collides with the scattering component in the medium, when the light is scattered in directions other than the incident direction $\vec{\omega}$, the light traveling in the direction $\vec{\omega}$ is attenuated. $\mathrm{L}$ in this phenomenon is shown in equation (1).

$$
d L(x, \vec{\omega})=-\sigma_{s}(x) L(x, \vec{\omega}) d s
$$

Here, $L(x, \vec{\omega})$ represents the intensity of incident light, and $\sigma_{s}(x)$ is called the scattering coefficient. The scattering coefficient represents how much light is scattered in directions other than the incident direction per minute distance.

As light travels in the medium, as shown by the arrow in the middle of Fig. 1, the amount of light is attenuated by the light being absorbed by the medium. At this time, $d L(x, \vec{\omega})$ is as shown in equation (2).

$$
d L(x, \vec{\omega})=-\sigma_{a}(x) L(x, \vec{\omega}) d s
$$

Here, $\sigma_{a}(x)$ is an absorption coefficient and represents the ratio of how much light is absorbed per minute distance.

The attenuation of incident light defined by Eq. (1) and (2) can be summarized as Eq. (3).

$$
d L(x, \vec{\omega})=-\sigma_{t}(x) L(x, \vec{\omega}) d s
$$

Here $\sigma_{t}$ is an extinction coefficient and is defined as the sum of the absorption coefficient and the scattering co- efficient. If $\sigma_{t}$ is a constant, it becomes a very simple exponential function, and the light when advancing in the medium from the position $x$ by the distance $d, L(x+$ $d, \vec{\omega})$ is as shown in Eq. (4).

$$
L(x+d, \vec{\omega})=e^{-\sigma_{t} d} L(x, \vec{\omega})
$$

\subsection{Light Scattering in Participating Media}

As shown by the lower arrow in Fig. 1, light incident from a direction other than $\vec{\omega}$ at position $x$ in the medium scatters in the $\vec{\omega}$ direction, and the light in the $\vec{\omega}$ direction increases. The amount of light increase in the $\vec{\omega}$ direction is as shown in Eq. (5).

$$
\begin{aligned}
& d L(x, \vec{\omega}) \\
& =\sigma_{s}(x) \int_{s^{2}} f_{p}\left(x, \vec{\omega}^{\prime}, \vec{\omega}\right) L(x, \vec{\omega}) d \vec{\omega}^{\prime} d s
\end{aligned}
$$

Here, $f_{p}\left(x, \vec{\omega}^{\prime}, \vec{\omega}\right)$ is called a phase function and we use the Henyey-Greenstein function ${ }^{(8)}$. The function characterizes the scattering direction of the participating media, and is mainly used to represent natural objects such as smoke and flames. $L(x, \vec{\omega})$ is light incident from a direction other than $\vec{\omega}$, and the total amount of light incident on the position $x$ and scattered in the $\vec{\omega}$ direction can be obtained by integration.

\subsection{Rendering of Reference Images}

We render an image by volume rendering for each frame. The rendering of each image follows the reflection and refraction of light in the same way as ray tracing. Also, in order to consider the media involved, processing of light attenuation and increase is added.

The scene to be rendered does not change, but the medium moves every frame. Therefore, in order to accurately represent the medium, rendering for each frame is required.

\section{Methods}

At first, we render one still image (hereinafter referred to as a base image) without considering the medium. Next, the DLI is combined to generate an image by shortening the creation time compared to the volume rendering image. In addition, by making illusionary smoke move, we express smoke movement similar to volume rendering animation.

DLI is luminance information that changes with time, and a value from 0 to 1 is stored for each pixel. In this 


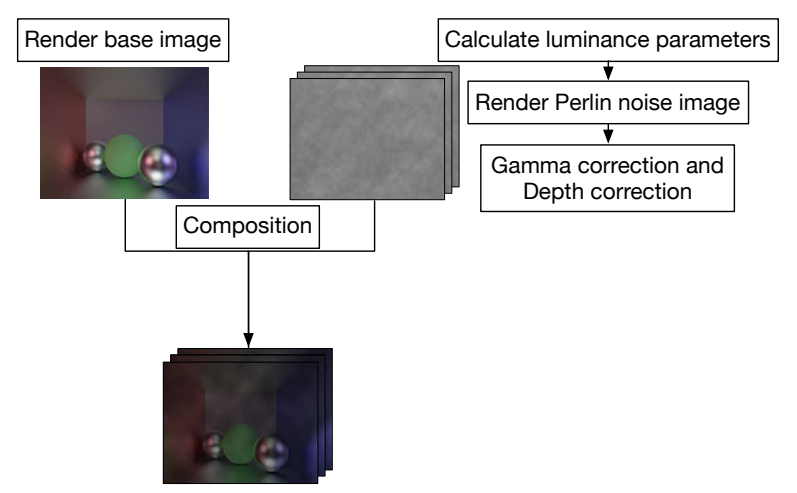

Fig. 2. Overview of our method.

method, no motion is given to objects in the reference image in order to reproduce the motion of the smoke. However, in the method of creating DLI presented in the paper ${ }^{(2)}$, motion is applied to objects in the image, so this method uses a different creation method. Figure 2 shows the outline of the proposed method.

\subsection{Rendering of Base Image}

Multiplexed Metropolis Light Transport (MMLT) ${ }^{(9)}$ is used to render the reference image. MMLT is a kind of ray tracing method, combining Markov chain Monte Carlo method and multi-focused sampling to construct a path very efficiently. The path connects the light source to the light entering the camera, and a CG image is generated by calculating the amount of light entering the camera for each path.

Path construction and light quantity calculation use Markov chain Monte Carlo method. In addition, multi-weighted sampling is used to reduce the variance of the amount of light that occurs during Monte Carlo calculation. In the rendering of the reference image, the scattering coefficient and the absorption coefficient are 0 , because the medium is not considered. As a result, light attenuation and increase by the medium will not occur.

\subsection{Generation for Dynamic Luminance Information}

The DLI is luminance information that changes with time, and a real value from 0 to 1 is stored for each pixel.

The central image group in Fig. 2 is the image of the DLI, and the luminance information is arranged in frames in chronological order. The value of each luminance information is calculated by brightness parameter, Perlin noise, gamma correction and distance correction.

\subsubsection{Luminance Determination}

We calculate a luminance $L$ as follows. First, a volume rendering image to be a reference is created, the extinction coefficient used is the reference coefficient $\sigma_{t}$, and the luminance average of the first frame is $L_{s}$. Next, input the extinction coefficient $\sigma_{t}{ }^{\prime}$ of the animation you want to create. The luminance is determined from the ratio $r$ of $\sigma_{t}$ and $\sigma_{t}{ }^{\prime}$. Equation (6) shows the ratio $r$ and the luminance $L$.

$$
\begin{gathered}
r=\frac{\sigma_{t}^{\prime}}{\sigma_{t}} \\
L=L_{s}\left(\frac{2}{3}\right)^{r-1}
\end{gathered}
$$

\subsubsection{Perlin Noise Generation}

Perlin Noise ${ }^{(10)}$ is a texture generation technique for realistic representation of the $\mathrm{CG}$, can generate a spatially and temporally varying value. To generate DLI, we use Perlin noise with coordinates and time as arguments. We use the vertical and horizontal coordinates of the reference image as coordinates, and also use the frame number of DLI as time.

In Perlin noise, noise whose frequency or amplitude is changed is called an octave. We use octave Perlin noise, which is Perlin noise in which multiple octaves are combined. We show the function $N()$ of octave Perlin noise in Eq. (7).

$$
N\left(\frac{x}{d_{1}}, \frac{y}{d_{1}}, \frac{f}{d_{2}}, O, P\right)
$$

Here, $x$ and $y$ are the coordinates of a pixel in the reference image, $f$ is a frame number, $d_{1}$ is a parameter that controls the spatial frequency of the noise, and $d_{2}$ controls the temporal frequency. $O$ represents the number of octaves, and $P$ represents the influence of octaves.

\subsubsection{Gamma correction and distance correction}

We use gamma correction and distance correction to enhance the smoke-like expression. As for gamma correction, we adjust the contrast of the image by changing the brightness of the image non-linearly. In our implementation, we use gamma correction to darken the contrast overall and make the smoke more pronounced.

On the other hand, in order to express the smoke density difference due to the depth in the space, we perform distance correction to make the center of the image darker. We use a Gaussian function to brighten as the distance from the screen center increases. The reason for using the Gaussian function is that many events in the natural world 
follow a normal distribution, so we thought that it might be closer to natural smoke.

\subsection{Composition of Dynamic Luminance Information}

We calculate the DLI by performing gamma correction and distance correction, and multiplying the brightness parameter for the each pixel of Perrin noise.

The final image is obtained by combining the DLI of each frame with the reference image. In compositing, each pixel of the reference image is multiplied by the value of the DLI stored in the corresponding pixel. This compositing process is performed on all the frames to obtain an image that makes the smoke move perceptible.

\section{Experiments}

Two evaluation experiments were conducted by creating six types of animation (hereinafter referred to as target animation) by volume rendering and animation (hereinafter referred to as proposed animation) by the proposed method.

One is quantitative evaluation using two image quality evaluation indicators, Structural Similarity (SSIM) ${ }^{(11)}$ and Multi Scale Structural Similarity (MS-SSIM) ${ }^{(12)}$. The image quality evaluation index quantifies how similar two types of images and animations are. With the target animation as the correct animation, it is possible to evaluate how similar the composite animation is to the correct animation as a numerical value.

The other is subjective evaluation using questionnaire. The subject looks at the two pictures lined up and asks which one is more smoked. By displaying the left side image always same, it is possible to evaluate the correspondence between the images by the two methods.

\subsection{Evaluation Images}

We use a total of 12 types of images for evaluation: 6 target images and 6 images generated by our method. Let the target animation be A, B, C, D, E, F, and the animation generated by our method approximated to each target animation be A', B', C', D', E', F'. Each image has 100 frames, and the frame rate is 6 fps.

Rendering an image using MMLT takes a lot of time. We set the rendering time for each frame of the target animation to 600 seconds in order to render the animation with an image quality that causes no noise in the animation. Since the target animation needs to render all the frames, the time to generate one animation is $6.0 \times 10^{4}$ seconds.

The generation time of the target image is the sum of the rendering time of the reference image, the generation time of the DLI, and the composition time of the two images. The rendering time of the reference image is also set to 600 seconds in accordance with the rendering time of the target image. The generation and combination of DLI need very short time and takes less than 30 seconds.

In order to create a proposal animation corresponding to each target animation, the extinction coefficient of the target animation is created as an input. Table 1 shows the scattering coefficient, the absorption coefficient, and the annihilation coefficient, which is the sum of two coefficients, used to generate the image used in the experiment. The animation used as the reference of the brightness parameter is A. Figure 3 shows the reference image for combining the DLI and the image of the first frame of A, A', F, and $\mathrm{F}^{\prime}$.

The CPU of the PC used for image creation is an Intel Core i7-6700 with 16Gbytes of memory, and the GPU is an NVIDIA GeForce GTX 960.

\subsection{Evaluations by Image quality evaluation indica- tors}

With the target animation as the correct answer, we used SSIM and MS-SSIM to evaluate how far the proposed animation is from the correct answer.

SSIM is an image quality evaluation index for images and animations, and MS-SSIM is a method to obtain

Table. 1. Coefficients for rendering images

\begin{tabular}{|c|c|c|c|}
\hline $\begin{array}{l}\text { Image } \\
\text { pair }\end{array}$ & $\begin{array}{c}\text { Scattering } \\
\text { factor }\end{array}$ & $\begin{array}{c}\text { Absorption } \\
\text { factor }\end{array}$ & $\begin{array}{c}\text { Extinction } \\
\text { coefficient }\end{array}$ \\
\hline $\mathrm{A}^{\cdot} \mathrm{A}^{\prime}$ & 0.010 & 0.015 & 0.025 \\
\hline $\mathrm{B} \cdot \mathrm{B}^{\prime}$ & 0.012 & 0.018 & 0.030 \\
\hline $\mathrm{C} \cdot \mathrm{C}^{\prime}$ & 0.014 & 0.021 & 0.035 \\
\hline $\mathrm{D} \cdot \mathrm{D}^{\prime}$ & 0.016 & 0.024 & 0.040 \\
\hline $\mathrm{E} \cdot \mathrm{E}^{\prime}$ & 0.018 & 0.027 & 0.045 \\
\hline $\mathrm{F} \cdot \mathrm{F}^{\prime}$ & 0.020 & 0.030 & 0.050 \\
\hline
\end{tabular}

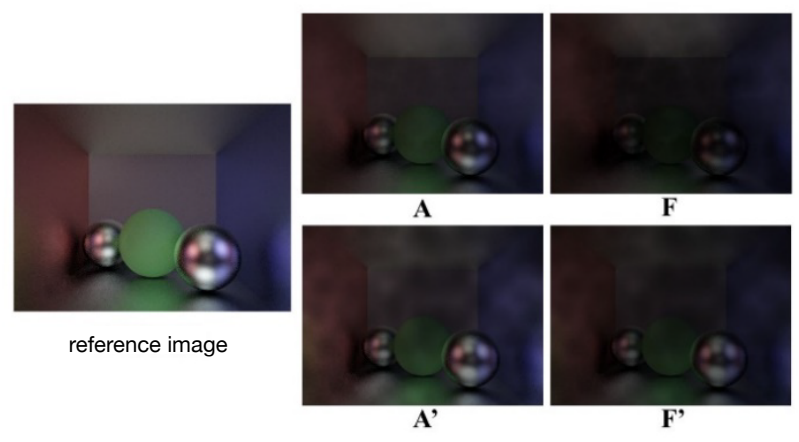

Fig. 3. Reference image and evaluated images (upper: target images, lower: proposed images) 
SSIM while gradually blurring the image. Both indicators can be evaluated near human visual characteristics.

\subsubsection{SSIM and MS-SSIM}

SSIM numerically shows how similar two images are. First, local regions are selected from the original image and the comparison image, and local SSIM, which is the similarity in the region, is determined. Next, the local region is shifted little by little, and the average of SSIMs obtained in each region is the final SSIM value. By finding the SSIM value while shifting the local region, it is possible to capture local differences in the image, and results similar to human visual characteristics can be obtained. Equation (8) shows the calculation formula of SSIM.

$$
I=\frac{\left(2 \mu_{x} \mu_{y}+c_{1}\right)\left(2 \sigma_{x y}+c_{2}\right)}{\left(\mu_{x}^{2}+\mu_{y}^{2}+c_{1}\right)\left(\sigma_{x}^{2}+\sigma_{y}^{2}+c_{2}\right)}
$$

Here, $\mu$ is the average of pixel values in the local region, and $\sigma$ is the standard deviation of the pixel values. The subscript $x$ represents the original image, and $y$ represents the comparison image. $\sigma_{x y}$ means covariance, $c_{1}$ and $c_{2}$ are constants.

On the other hand, MS-SSIM blurs the original image and the comparison image in stages, and finds the SSIM value at each stage. The obtained SSIM values are weighted to obtain the final MS-SSIM value. By using graded evaluation values, it has been shown to function as well as or better than $\operatorname{SSIM}^{(13)}$.

\subsubsection{Results}

Figure 4 shows the evaluation values of SSIM and

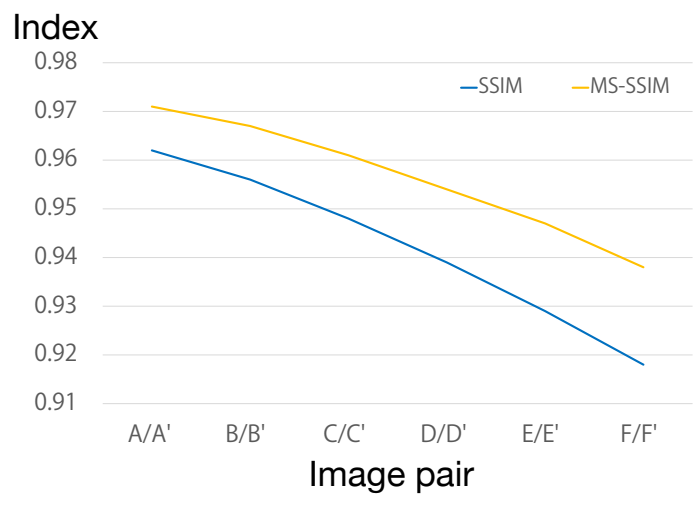

Fig. 4. SSIM and MS-SSIM for image pairs.
MS-SSIM. In the case of animation evaluation, the evaluation value is obtained for each frame and the average value is used.

Each evaluation index outputs a real value of 0 to 1 , and the closer to 1 , the more similar the two images are. The target value is 0.95 or more, which is the value at which human eyes can't recognize the difference.

From the two evaluation results, the image pair of A - A' and B·B' exceeded the target value. For C.C' and D'D', only MS-SSIM exceeded the target value. It can be seen that the evaluation value decreases as the extinction coefficient rises for either index.

\subsection{Evaluations by Questionnaire}

We conducted the same questionnaire as conducted in the paper ${ }^{(2)}$ to compare the two animations. The subjects were asked to view two images at the same time, and they were asked to answer which one looks more like smoke, right or left. The subjects were eight males and two females, who were 21-25 years old.

\subsubsection{Experimental Method}

The target animation or composite animation are displayed on the left and right on a display, and the subject looked at it. We always display the target animation $\mathrm{C}$ on the left. The image on the right is one of 12 types of animations including $\mathrm{C}$. The subject doesn't know that the animation on the left is always the same image $\mathrm{C}$.

Since the order in which the animations are displayed is random and the same animation is not selected, there is no duplication in the animation seen by the subject. The subject gives a total of 12 answers.

The subject sits at a distance of about $70 \mathrm{~cm}$ from the display. There is no limit to the displayed time of one animation, and the displaying continues until the subject finished answering.

\subsubsection{Results}

We define the value as 1 if a subject selects the right and the value as 0 if a subject selects the left for the displayed animation. Figure 5 shows two graphs of the average values. 


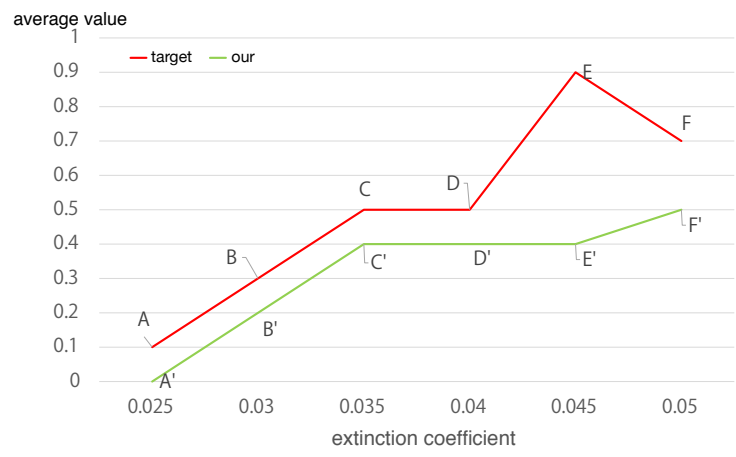

Fig. 5. Extinction coefficient and average value of questionnaire responses

The image on the left is always $\mathrm{C}$, so if it is less than 0.5 , it feels less like smoke than $\mathrm{C}$, and if it is greater than 0.5 Indicates that you feel like smoke than C. It can be seen that the average value of the target image tends to increase from A to E. However, F has a lower value than E. On the other hand, in the proposed animation, the values tended to increase from A' to F'. However, the same value was taken from $C^{\prime}$ to $E^{\prime}$ and no upward trend was observed in this section.

The proposed animation did not exceed the target animation for any extinction coefficient. However, for the extinction coefficient from 0.025 to 0.04 , the graph of the proposed image and the target image had the same shape with a difference of 0.1 .

In addition, t-tests were performed with the confidence level set to $95 \%$ for the responses of the proposed animation and the target animation for each extinction coefficient. A significant difference was found in the image pair with an extinction coefficient of 0.045 , and no other differences were found.

\section{Discussion}

Figure 4 shows that the image quality evaluation index decreases with both SSIM and MS-SSIM as the extinction coefficient increases.

In SSIM, A and B, and in MS-SSIM, A to D exceeded the value 0.95 , which is considered to be indistinguishable to the human eye. From this, it can be said that the animation of the proposed method and the animation of the existing method are quantitatively similar in the range of the extinction coefficient of this time. However, it is thought that the evaluation value declines with the increase of the extinction coefficient from the rough form of the graph. It is considered that the evaluation value decreased because the smoke density increased with the increase of the extinction coefficient and the difference between the pixel values of the pixels with and without smoke increased.

Figure 5 shows that the average value of the questionnaire evaluation of the proposed animation increases with the increase of the extinction coefficient. The average value of the target image also showed an upward trend, but from the extinction coefficient of 0.045 . The value decreased to 0.05 . Also, the average value of the proposed animation did not exceed the average value of the target animation. From this, it can be said that the proposed animation did not exceed the perceptual quality of the target animation.

The average of the responses of the target animation and the proposed animation remained constant at intervals between 0.025 and 0.04 . Also, since no significant difference was found in the coefficients of the same interval in the $t$ test, it can be said that there was no difference in the subject's perception. From these facts, it can be said that the animation created by the proposed method maintains the same perceptual quality as the target animation at extinction coefficients of 0.025 to 0.04 .

\section{Conclusions}

We have presented an image creation method that gives illusionary smoke movement using DLI. Our method is about 100 times faster than using volume rendering to render smoke animations.

We also have experimented two evaluations of image quality evaluation index and questionnaire using the created animations. From the experiments with the image quality evaluation index, we have found that the images by the two methods are similar in quantitative terms when the extinction coefficient is low. Also, from the result of the experiment by the questionnaire and the result of $t$ test, we have found that the images of the two methods have the same level of perceptual quality in the extinction coefficient such that the smoke concentration becomes thinner.

From the above, it is possible to rapidly create low density smoke animation without degrading the perceived quality as compared to volume rendering.

In the future, we would like to propose a method to create images with similar perceived quality for volume rendering images with various extinction coefficients. For this purpose, it is necessary to improve the method of generating DLI. For example, in the generation of Perlin 
noise, the output result is highly dependent on the parameters. By changing the parameters to be used by the extinction coefficient of the volume rendering animation, it is considered that the perceptual quality of the animation can be further improved.

\section{References}

(1) Eric P. Lafortune and Yves D. Willems : "Rendering participating media with bidirectional path tracing", Proceedings of Rendering Techniques '96, pp. 91-100, 1996

(2) Takahiro Kawabe, Taiki Fukiage, Masataka Sawayama, and Shin'ya Nishida : "Deformation lamps: A projection technique make static objects perceptually dynamic", ACM Transactions on Applied Perception, vol. 13, no. 2, 2016

(3) Yusuke Chiba and Takafumi Koike : "Approximation of smoke volume rendering animation using brightness kinetic information", The 80th National Convention of IPSJ, 3ZB-06, 2018 (In Japanese)

(4) Ronald Fedkiw, Jos Stam, and Henrik Wann Jensen : "Visual simulation of smoke", Computer Graphics SIGGRAPH '01 Proceedings, pp. 15-22, 2001

(5) Albert Chern, Felix Knoppel, Ulrich Pinkall, Peter Schröder, and Steffen Weißmann : "Schrödinger's smoke", ACM Transactions on Graphics, vol. 35, no. 4, pp. 1-13, 2016

(6) Makoto Okabe, Ken Anjyo, and Rikio Onai : "Creating fluid animation from a single image using video database", Computer Graphics Forum, vol. 30, no. 7, pp. 1973-1982, 2011

(7) James T. Kajiya and Brian P. Von Herzen : "Ray tracing volume densities", Computer Graphics SIGGRAPH'84 Proceedings, vol. 18, no. 3, pp. 165-174, 1984

(8) L. G. Henyey and J. L. Greenstein : "Diffuse radiation in the Galaxy", Astrophysical Journal, vol. 93, pp. 70-83, 1941

(9) Ken Perlin : "Improving noise", ACM Transactions on Graphics, vol. 21, no. 3, pp. 681-682, 2002

(10) Toshiya Hachisuka, Anton S. Kaplanyan, and Carsten Dachsbacher : "Multiplexed Metropolis Light Transport”, ACM Transactions on Graphics, vol. 33, no.4, pp. 1-10, 2014
(11)Zhou Wang, Alan Conrad Bovik, Hamid Rahim Sheikh, and Eero P. Simoncelli : "Image quality assessment: from error visibility to structural similaruty", IEEE Transactions on Image Processing, vol. 13, no.4, pp. 600-612, 2004

(12)Zhou Wang, Eero P. Simoncelli, and Alan Conrad Bovik : "Multi-scale structural similarity for image quality assessment", Conference Record of the Thirty-Seventh Asilomar Conference on Signals, Systems and Computers, vol. 2, no. 5, pp. 1398-1402, 2003

(13) Jacob Søgaard, Lukáš Krasula, Muhammad Shahid, Dogancan Temel, Kjell Brunnström, and Manzoor Razaak : “Applicability of Existing Objective Metrics of Perceptual Quality for Adaptive Video Streaming", Image quality and System Performance XIII on Electronic Imaging, vol. 13, pp. 1-7, 2016 